\title{
GENETIC VARIATION IN DIPLOID DACTYLIS III. PANICLE, SPIKELET AND FLORET CHARACTERS
}

\author{
P. F. PARKER \\ Hartley Botanical Laboratories, The University, Liverpool*
}

Received 7.x.68

\section{Introduction}

IN this final paper of a series describing the pattern of genetic variation to be found in some subspecies populations of diploid Dactylis, the $9 \times 9$ diallel cross previously described (Parker, $1968 a, b$ ) is analysed for a series of panicle characters, some of which have been replicated within the plant to study the reliability of the genetic information provided by a single measurement.

\section{Materials AND methods}

The same subspecies numbers as before are used in the tables and graphs (fig. 1) and the characters considered are:

(i) Panicle length (mm.).

(ii) Number of florets per spikelet.

(iii) Spikelet length ( $\mathrm{mm}$.).

(iv) Lemma length. Measured on a Prior binocular microscope; micrometer units used in table $=\mathrm{mm} . \times 2$.

(v) Lemma awn length, measured as (iv).

(vi) Lemma apex notch depth, measured as (iv).

The first three panicles to emerge on each plant were measured separately and analysed as genotype replicates (i.e. intra-plant replicates) in order to estimate the extent of variation in gene expression due to environmental and developmental changes within the plant. The two characters, florets per spikelet and spikelet length, were similarly treated, using two genotype replicates.

The extended Hayman's analysis of variance for these three characters is similar to that used by Broadhurst and Jinks (1966). Analysis of the results summed over genotype replicates gives the variation within samples. For the main effects items to be significant, they must be significantly greater than all three interaction items, however as the block interactions were the largest in all cases, main effects were tested against these. The block and genotype replicate interactions were tested against the appropriate residual items in each case.

The variance $(V r)$ covariance $(W r)$ analysis developed by Jinks (1954) was also used in the genetic assessment of the populations. Both this analysis and the parental means were further analysed using the method proposed by Allard (1956); this method gives an assessment of the stability of three parameters, namely, additive effects, dominance effects and epistatic effects.

* Present Address, Botany Department, University of Leicester. 
The analysis of parental means tests for additive genetic effects; constancy of these additive components of variation can be detected by the parents $X$ genotype replicates item, significance indicating interaction, and nonsignificance indicating constancy of expression in the different genotype replicates.

A good test for the constancy of additive components of variation being available from analysis of parental means, this component is minimised in the analysis of $W r$ and $V r$ by dividing them by the variance of the parents $(V p)$ occurring in the same block. This improves the prospects of detecting dominance $\times$ genotype replicate interactions by placing comparisons between them on similar scales. The $W^{\prime} r / W r$ graph is also used to detect the order of dominance of the parents, it is much less affected by genetic disturbances than the $W r / V r$ graph and virtually undisturbed by the level of inbreeding (Hayman, 1958). The $W^{\prime} r / W r$ graph differs from the $W r / V r$ however, in that it is more obviously affected by asymmetry of gene distribution, and indicates this whether the genes are correlated or not (Hayman, 1958). However, as both graphs are disturbed by both these effects to some extent it is not always possible to separate the causes of the disturbance with complete precision. With gene symmetry the regression of $W^{\prime} r$ on $W r$ is a straight line of slope +0.5 , when gene asymmetry occurs, parents with common genotypes will fall above the line of slope +0.5 , parents with different or relatively rare genotypes will fall below it. Both male and female points were put on the $W r / V r$ graphs when the Hayman's analysis indicated the presence of significant consistent reciprocal differences, but if the reciprocal regressions were not significantly different, the joint line was fitted. The environmental component of variation $\left(E_{2}\right)$ derived from the between blocks total interaction mean square from the Hayman's analysis of variance, and appropriately calculated for $W r$ and $\operatorname{Vr}$ (Jinks, 1954) was also obtained, but only in two characters was the non-heritable variation large enough to noticeably alter the level of dominance indicated by the uncorrected $W r / V r$ graph. In these graphs the corrected ordinates and abscissae are put in as broken lines.

\section{(i) Panicle length}

\section{Results}

The mean lengths of each genotype replicate are presented in table 1, and Hayman's analysis of variance in table 2. The block interactions are homogeneous $\left(\chi_{(5)}^{2}=7.8 ; P=0.3\right)$ so individual main items were tested against the common interaction item $(B t)$. There are large and highly significant additive genetic differences between the populations (item $a$ ) and some dominance variation (item $b$ ). All three components of dominance are significant $\left(b_{1}\right)$ indicating the presence of directional dominance $\left(b_{2}\right)$ gene asymmetry, and $\left(b_{3}\right)$ inconsistent dominance interactions. The items of both block and replicate interactions are all significant (apart from Reps $\times c$ ), when tested against the items of the residual, the main replicates interaction is also significant. The replicates $\times a$ interaction stands out as being quite large, but this does not alter the significance of the main (a) item.

The $W r / V r$ graphs (fig. 1) show that the array points of all three genotype replicates fall on the same line, analysis of variance showed no significant differences between the regressions so a common line is fitted ( $b=$ $1 \cdot 142 \pm 0 \cdot 110 ; \mathrm{P}=0 \cdot 001)$, this cuts the $W r$ axis positively, indicating a low 
level of dominance. The correlation of parental means on $(W r+V r)$, $\left(r=0 \cdot 745^{*}\right)$ shows this to be directional for small panicle. The $W^{\prime} r / W r$ graph gives a similar distribution of points, the regression $(b=0.441 \pm$ $0.041 ; \mathrm{P}=0.001)$ is not significant from +0.5 .

TABLE 1

Mean panicle length (mm.) for the first (top line) second (centre line) and third (bottom line) genetic replicates

\begin{tabular}{|c|c|c|c|c|c|c|c|c|c|}
\hline & 1 & 2 & 3 & 4 & 5 & 6 & 7 & 8 & 9 \\
\hline \multirow[t]{3}{*}{1.} & 94.97 & $103 \cdot 21$ & $150 \cdot 26$ & $120 \cdot 49$ & $148 \cdot 01$ & $110 \cdot 91$ & $142 \cdot 35$ & 133.75 & $121 \cdot 40$ \\
\hline & $87 \cdot 9$ & $87 \cdot 4$ & $105 \cdot 14$ & 99. & 112. & & & & $101 \cdot 17$ \\
\hline & $87 \cdot 7$ & $77 \cdot 36$ & $94 \cdot 75$ & $86 \cdot 47$ & $102 \cdot 92$ & $91 \cdot 20$ & 83.57 & $76 \cdot 55$ & 87.94 \\
\hline \multirow[t]{3}{*}{2.} & $79 \cdot 08$ & $34 \cdot 01$ & $80 \cdot 72$ & $65 \cdot 85$ & $88 \cdot 26$ & $59 \cdot 26$ & $76 \cdot 15$ & 77.21 & $84 \cdot 71$ \\
\hline & $74 \cdot 7$ & $33^{\circ}$ & $62 \cdot 4$ & 57. & $78 \cdot 6$ & 57. & 46. & 57. & $59 \cdot 32$ \\
\hline & $61 \cdot 0$ & $34 \cdot 1$ & $55 \cdot 67$ & $51 \cdot 39$ & $79 \cdot 37$ & $52 \cdot 78$ & $46 \cdot 31$ & 03 & $54 \cdot 15$ \\
\hline \multirow[t]{3}{*}{3.} & & $82 \cdot 67$ & 118.54 & $156 \cdot 42$ & 167.5 & $138 \cdot 54$ & $127 \cdot 35$ & $142^{\circ} \cdot$ & 154.55 \\
\hline & $90 \cdot 24$ & $71 \cdot 60$ & 86.89 & 115.5 & 126 & 109 & $90^{\circ}$ & & 114.58 \\
\hline & $84 \cdot 38$ & $61 \cdot 47$ & 83.57 & $97 \cdot 01$ & 114.68 & $100 \cdot 28$ & $80 \cdot 29$ & $84 \cdot 14$ & $96 \cdot 76$ \\
\hline \multirow[t]{3}{*}{4.} & $108 \cdot 46$ & 68.06 & $160 \cdot 79$ & $110 \cdot 49$ & $156 \cdot 17$ & 133.43 & $126 \cdot 4$ & 137.62 & 144.09 \\
\hline & $95 \cdot 49$ & $58 \cdot 51$ & $129 \cdot 6$ & $99^{\circ}$ & 113. & 112. & 77. & $107 \cdot 70$ & $104 \cdot 52$ \\
\hline & $82 \cdot 26$ & $57 \cdot 22$ & $111 \cdot 43$ & $99 \cdot 34$ & $100 \cdot 61$ & $97 \cdot 28$ & $69 \cdot 12$ & $90 \cdot 80$ & $94 \cdot 13$ \\
\hline \multirow[t]{3}{*}{5.} & 1 & 7 & $74 \cdot 9$ & 6. & 187 & 2 & 144 & 168 & 166 \\
\hline & 1 & 8 & 12 & 1 & & & 102 & & \\
\hline & $101 \cdot 53$ & $78 \cdot 64$ & $115 \cdot 10$ & $104 \cdot 29$ & $138 \cdot 35$ & $125 \cdot 82$ & $90 \cdot 80$ & $100 \cdot 75$ & $116 \cdot 07$ \\
\hline \multirow[t]{3}{*}{6.} & 118.85 & $6 \cdot($ & $125 \cdot 2$ & $17 \cdot 6$ & 158 & 145 & 124 & 156 & 148.79 \\
\hline & $95 \cdot 74$ & 6 & $95 \cdot 6$ & $107 . \zeta$ & 131 & 122 & 89 & 101 & 114.55 \\
\hline & & 64.08 & $86 \cdot 90$ & $101 \cdot 41$ & 118.62 & $123 \cdot 26$ & $81 \cdot 35$ & 87.60 & 93.93 \\
\hline \multirow[t]{3}{*}{7.} & 133.28 & $83 \cdot 76$ & 120 & 133. & 154 & 112 & 102 & 137 & \\
\hline & $103 \cdot 2$ & 6 & $81 \cdot$ & $90 \cdot 4$ & 118.06 & 79. & 67 & 94 & $108 \cdot 89$ \\
\hline & $84 \cdot 87$ & $55 \cdot 33$ & $66 \cdot 45$ & $89 \cdot 16$ & $96 \cdot 39$ & $75 \cdot 60$ & $55 \cdot 82$ & $80 \cdot 45$ & $87 \cdot 12$ \\
\hline & 141.04 & 9 & 139. & 1 & & & 123 & & $175 \cdot 79$ \\
\hline & & & & & & & & & $99 \cdot 94$ \\
\hline & 86.96 & 49.33 & $70 \cdot 59$ & 95.98 & $104 \cdot 45$ & $96 \cdot 86$ & 82.03 & 68.92 & $93 \cdot 11$ \\
\hline & $127 \cdot 58$ & & & & & & $128^{\circ}$ & & \\
\hline & $88 \cdot 14$ & & $104 \cdot 2$ & 104.9 & $128 \cdot 92$ & & $95 \cdot 24$ & $110 \cdot 42$ & $83 \cdot 23$ \\
\hline & $81 \cdot 01$ & $57 \cdot 07$ & $78 \cdot 70$ & $90 \cdot 17$ & $106 \cdot 50$ & $92 \cdot 21$ & $81 \cdot 42$ & $94 \cdot 67$ & $2 \cdot 84$ \\
\hline
\end{tabular}

Analysis of parental means (table $3 a$ ) gives a highly significant mean square for parents, indicating that they have different additive and/or epistatic effects. The significant genotype replicates mean square shows that the expression of the additive genes differed between replicates, this is no doubt related to the different times of initiation, and different growth periods of the panicle primordia. The mean square for the parents $\times$ genotype replicates interaction is large and highly significant, this provides evidence for the interaction of additive and/or additive $\times$ additive gene effects with 
genotype replicates. There is also a significant interaction between parents and blocks. The order of parental panicle size is:

Large

Small

$\begin{array}{rrrrrrrrrrr}\text { G.R. } & \text { I } & 5 & 6 & 9 & 8 & 4 & 1 & 3 & 7 & 2 \\ & \text { II } & 5 & 6 & 8 & 4 & 3 & 1 & 9 & 7 & 2 \\ & \text { III } & 5 & 6 & 4 & 3 & 8 & 9 & 1 & 7 & 2\end{array}$

TABLE 2

Combined Haymans analysis of variance including genotype replicates, for panicle length, number of forets per spikelet, and spikelet, and spikelet length
(a) Panicle length
(b) No. of Florets
(c) Spikelet length

Item

$a$
$b_{1}$
$b_{2}$
$b_{3}$
$b$
$c$
$d$

Total

Blocks

$B a$

$B b_{1}$

$B b_{2}$

$B b_{3}$

$B b$

$B c$

$B d$

$B$ total

Total

Replicates

$R a$

$R b_{1}$

$R b_{2}$

$R b_{3}$
$R b$

$R c$

$R d$

$R$ total

Blocks $x$

Replicates

$B \times R \times a$

$B \times R \times b$

$B \times R \times b_{2}$

$B \times R \times b_{3}$

$B \times R \times b$

$B \times R \times c$

$B \times R \times d$

$B \times R$ total

Total

Grand total 726
V.R.

D.F. M.S.

$10 \cdot 43$

6.04

$1 \cdot 18$

$2 \cdot 16$

$2 \cdot 05$

0.77

0.57

403.40

$5 \cdot 52 * * * 36$

I. 66

28

80

80

$22920 \cdot 04$

16

2

16

54
72

16

56

160

242

2
16
2
16
54
72
16
56

$230 \cdot 65$

$795 \cdot 11$

111.52

$201 \cdot 61$

$202 \cdot 63$

290.92

$282 \cdot 51$

$242 \cdot 22$

$\begin{array}{lr}2 \cdot 68 & 2 \\ 2 \cdot 10^{*} & 16 \\ 15 \cdot 81^{*} & 2 \\ 2 \cdot 71^{* *} & 16 \\ 2 \cdot 74^{* *} & 54 \\ 3 \cdot 08^{* * *} & 72 \\ 4 \cdot 77^{* * *} & 16 \\ 6 \cdot 31^{* * *} & 56 \\ & 160\end{array}$

$1 \cdot 7$

0.34

$0 \cdot 05$

$0 \cdot 36$

0.39

0.38

$0 \cdot 26$

0.38

0.36

242

$117061 \cdot 64$
$2399 \cdot 22$
$393 \cdot 51$
$162 \cdot 73$
$174 \cdot 48$
$177 \cdot 95$
$78 \cdot 55$
$70 \cdot 14$
$352 \cdot 40$

107.53** 1

$7 \cdot 13$

0.07

0.02

0.05

0.03

0.03

0.02

0.03

0.03

$18 \cdot 35$

5.0251

0.0429

0.0567

0.0236

$1.42 \quad 0.0141$

0.0174

0.0170

$1 \cdot 19$

0.0193

$0 \cdot 0206$

0.3313

0.0296

0.0242

$0 \cdot 0272$

0.0388

0.0358

0.0348

0.0141

60.93

44.78

$62 \cdot 30$

0.05

0.02

0.04

0.0275
$15 \cdot 17$

1. 45

$2 \cdot 34$

I.3/
V.R.

7.90 ***

$1 \cdot 12$

I. 42

$2 \cdot 36$

$6 \cdot 77^{* * *}$

3.52

$11.02 * * *$

$5 \cdot 34 * * *$

$6 \cdot 27 * * *$

$6 \cdot 28^{* * *}$

$24 \cdot 31^{* * *}$

484

${ }^{*} \mathrm{P}=0.05 ; * * \mathrm{P}=0.01 ; * * * \mathrm{P}=0.001$ 

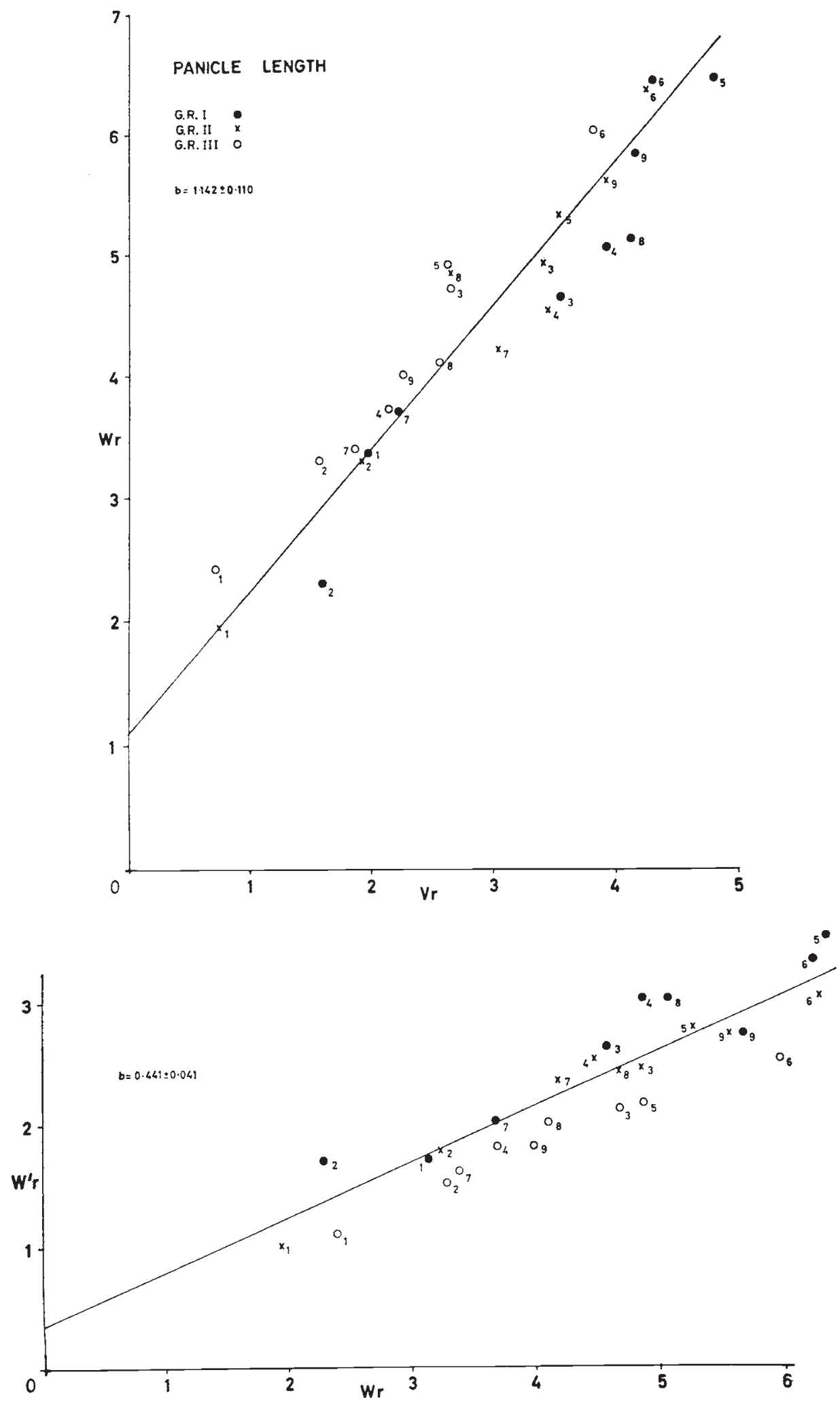

Fig. 1.-Wr/Vr and $W^{\prime} r / W r$ graphs for panicle length. Numbers are as follows; 1 smithii; 2. juncinella; 3 . lusitanica; 4. aschersoniana; 5 . parthiana; 6. himalayensis; 7. ibizensis; 8. santai; 9. castellata. 
The most variable parents appear to be castellata (9), lusitanica (3) and santai (8), they are also the ones that change position most on the $\mathrm{Wr} / \mathrm{Vr}$ graph.

In the analysis of variance of $W r$ and $V r$ (table $3 \mathrm{~b}$ ), only three mean squares are of interest; the significance of that for genotype replicates suggests

TABLE $3 a$

Analysis of variance of parental means

\begin{tabular}{lrrc}
\multicolumn{1}{c}{ Item } & D.F. & \multicolumn{1}{c}{ M.S. } & V.R. \\
Parents & 8 & $10584 \cdot 74$ & $189 \cdot 41 * * *$ \\
Blocks & 2 & $156 \cdot 40$ & $2 \cdot 80$ N.S. \\
Genotype replicates & 2 & $9385 \cdot 28$ & $167 \cdot 95^{* * *}$ \\
Blocks $\times$ Gen. reps. & 4 & $177 \cdot 51$ & $3 \cdot 18^{*}$ \\
Parents $\times$ Gen. reps. & 16 & $632 \cdot 59$ & $11 \cdot 32^{* * *}$ \\
Parents $\times$ blocks & 16 & $197 \cdot 54$ & $3 \cdot 53^{* *}$ \\
Error & 32 & $55 \cdot 88$ & \\
Total & 80 & &
\end{tabular}

TABLE $3 b$

Analysis of variance of $\mathrm{Wr}$ and $\mathrm{Vr}$ for panicle length

\begin{tabular}{lrrc}
\multicolumn{1}{c}{ Item } & D.F. & M.S. & V.R. \\
Arrays & 8 & $20 \cdot 78$ & $36 \cdot 15^{* * *}$ \\
Genetic replicates & 2 & $9 \cdot 51$ & $16 \cdot 54^{* * *}$ \\
Dominance & 1 & $59 \cdot 84$ & $104 \cdot 13^{* * *}$ \\
G.r's $\times$ arrays & 16 & $1 \cdot 31$ & $2 \cdot 28 * *$ \\
G.r's $\times$ dominance & 2 & $1 \cdot 10$ & $1 \cdot 92$ N.S. \\
Arrays $\times$ dominance & 8 & $0 \cdot 43$ & \\
G.r's $\times$ dom $\times$ arrays & 16 & $0 \cdot 09$ & \\
Error & 108 & $0 \cdot 57$ & \\
Total & 161 & &
\end{tabular}

$$
* \mathrm{P}=0 \cdot 05 ; * * \mathrm{P}=0 \cdot 01 ; * * \mathrm{P}=0 \cdot 001 .
$$

a change in mean dominance and/or the relative proportion of dominant and recessive effects within the plants. The mean square for genotype replicates $X$ arrays tests the constancy of the mean level of dominance for each parent over the three measurements, this is highly significant, indicating that the positions of the parental arrays altered relative to each other, either up or down the regression line, in different genotype replicates. The ranking of the parents in order of decreasing dominance was:

Dominant

Recessive

$\begin{array}{rrrrrrrrrrr}\text { G.R. } & \text { I } & 2 & 1 & 7 & 3 & 4 & 8 & 9 & 6 & 5 \\ & \text { II } & 1 & 2 & 7 & 8 & 4 & 3 & 5 & 9 & 6 \\ & \text { III } & 1 & 2 & 7 & 4 & 9 & 8 & 3 & 5 & 6\end{array}$

The genotype replicates $\times$ dominance mean square is non-significant, indicating that the regression lines for the three measurements on the same plant occupy the same positions. These results suggest that both additive gene effects and dominance relationships among various parental populations were affected by intra-plant variability, however, the shifts in dominance that occurred among individual parental populations varied at random 
around the mean so that the mean level of dominance over all arrays was not altered.

\section{(ii) Number of florets per spikelet}

The two genotype replicates were taken from different areas of the panicle, one from the lower part of a basal glomerulus, the other from the same area of a glomerulus taken from the centre of the head.

\section{TABLE 4}

Mean number of florets per spikelet for the first (top line) and second genetic replicates

\begin{tabular}{|c|c|c|c|c|c|c|c|c|c|}
\hline & 1 & 2 & 3 & 4 & 5 & 6 & 7 & 8 & 9 \\
\hline 1. & $\begin{array}{l}5 \cdot 39 \\
5 \cdot 51\end{array}$ & $\begin{array}{l}4 \cdot 22 \\
4 \cdot 58\end{array}$ & $\begin{array}{l}6 \cdot 53 \\
6 \cdot 69\end{array}$ & $\begin{array}{l}6.03 \\
6 \cdot 08\end{array}$ & $\begin{array}{l}7 \cdot 22 \\
7 \cdot 33\end{array}$ & $\begin{array}{l}6 \cdot 25 \\
6 \cdot 36\end{array}$ & $\begin{array}{l}6 \cdot 42 \\
6 \cdot 42\end{array}$ & $\begin{array}{l}5 \cdot 97 \\
6 \cdot 30\end{array}$ & $\begin{array}{l}4 \cdot 44 \\
4 \cdot 53\end{array}$ \\
\hline 2. & $\begin{array}{l}4 \cdot 69 \\
4 \cdot 78\end{array}$ & $\begin{array}{l}4 \cdot 20 \\
4 \cdot 51\end{array}$ & $\begin{array}{l}5 \cdot 75 \\
5 \cdot 92\end{array}$ & $\begin{array}{l}5 \cdot 58 \\
6 \cdot 17\end{array}$ & $\begin{array}{l}5 \cdot 60 \\
5 \cdot 62\end{array}$ & $\begin{array}{l}5 \cdot 44 \\
5 \cdot 78\end{array}$ & $\begin{array}{l}5 \cdot 14 \\
5 \cdot 56\end{array}$ & $\begin{array}{l}5 \cdot 05 \\
5 \cdot 30\end{array}$ & $\begin{array}{l}4 \cdot 58 \\
4 \cdot 92\end{array}$ \\
\hline 3. & $\begin{array}{l}6 \cdot 25 \\
6 \cdot 25\end{array}$ & $\begin{array}{l}5 \cdot 86 \\
6 \cdot 00\end{array}$ & $\begin{array}{l}5 \cdot 44 \\
6 \cdot 14\end{array}$ & $\begin{array}{l}5 \cdot 39 \\
5 \cdot 81\end{array}$ & $\begin{array}{l}5 \cdot 64 \\
5 \cdot 92\end{array}$ & $\begin{array}{l}5 \cdot 14 \\
5 \cdot 22\end{array}$ & $\begin{array}{l}5 \cdot 08 \\
5 \cdot 44\end{array}$ & $\begin{array}{l}5 \cdot 64 \\
5 \cdot 69\end{array}$ & $\begin{array}{l}4 \cdot 61 \\
4 \cdot 81\end{array}$ \\
\hline 4. & $\begin{array}{l}5 \cdot 47 \\
6 \cdot 05\end{array}$ & $\begin{array}{l}5 \cdot 08 \\
5 \cdot 39\end{array}$ & $\begin{array}{l}6 \cdot 22 \\
6 \cdot 53\end{array}$ & $\begin{array}{l}5 \cdot 25 \\
5 \cdot 55\end{array}$ & $\begin{array}{l}6 \cdot 28 \\
6.68\end{array}$ & $\begin{array}{l}5 \cdot 14 \\
5 \cdot 67\end{array}$ & $\begin{array}{l}5 \cdot 26 \\
5 \cdot 69\end{array}$ & $\begin{array}{l}4 \cdot 87 \\
4 \cdot 89\end{array}$ & $\begin{array}{l}5 \cdot 09 \\
5 \cdot 26\end{array}$ \\
\hline 5. & $\begin{array}{l}5 \cdot 72 \\
5 \cdot 72\end{array}$ & $\begin{array}{l}5 \cdot 58 \\
6 \cdot 03\end{array}$ & $\begin{array}{l}4 \cdot 86 \\
5 \cdot 14\end{array}$ & $\begin{array}{l}6 \cdot 44 \\
6 \cdot 56\end{array}$ & $\begin{array}{l}6 \cdot 25 \\
6 \cdot 50\end{array}$ & $\begin{array}{l}5 \cdot 31 \\
5 \cdot 69\end{array}$ & $\begin{array}{l}6 \cdot 40 \\
6 \cdot 40\end{array}$ & $\begin{array}{c}5 \cdot 86 \\
5 \cdot 86\end{array}$ & $\begin{array}{l}5 \cdot 14 \\
5 \cdot 39\end{array}$ \\
\hline 6. & $\begin{array}{l}5 \cdot 64 \\
5 \cdot 86\end{array}$ & $\begin{array}{l}5 \cdot 30 \\
5 \cdot 61\end{array}$ & $\begin{array}{l}5 \cdot 50 \\
5 \cdot 83\end{array}$ & $\begin{array}{l}5 \cdot 64 \\
5 \cdot 85\end{array}$ & $\begin{array}{l}5 \cdot 38 \\
5 \cdot 67\end{array}$ & $\begin{array}{l}4 \cdot 99 \\
5 \cdot 25\end{array}$ & $\begin{array}{l}5 \cdot 28 \\
5 \cdot 54\end{array}$ & $\begin{array}{l}5 \cdot 36 \\
5 \cdot 58\end{array}$ & $\begin{array}{l}4 \cdot 53 \\
4 \cdot 64\end{array}$ \\
\hline 7. & $\begin{array}{l}6 \cdot 30 \\
6 \cdot 44\end{array}$ & $\begin{array}{l}5 \cdot 33 \\
5 \cdot 69\end{array}$ & $\begin{array}{l}4 \cdot 48 \\
4 \cdot 61\end{array}$ & $\begin{array}{l}4 \cdot 97 \\
5 \cdot 39\end{array}$ & $\begin{array}{l}5 \cdot 63 \\
5 \cdot 60\end{array}$ & $\begin{array}{l}5 \cdot 20 \\
5 \cdot 47\end{array}$ & $\begin{array}{l}4 \cdot 47 \\
4 \cdot 89\end{array}$ & $\begin{array}{l}5 \cdot 07 \\
5 \cdot 44\end{array}$ & $\begin{array}{l}4 \cdot 72 \\
4 \cdot 89\end{array}$ \\
\hline 8. & $\begin{array}{l}5 \cdot 64 \\
5 \cdot 89\end{array}$ & $\begin{array}{l}4 \cdot 92 \\
5 \cdot 05\end{array}$ & $\begin{array}{l}5 \cdot 83 \\
6 \cdot 03\end{array}$ & $\begin{array}{l}4 \cdot 83 \\
5 \cdot 07\end{array}$ & $\begin{array}{l}5 \cdot 67 \\
5 \cdot 92\end{array}$ & $\begin{array}{l}5 \cdot 53 \\
5 \cdot 78\end{array}$ & $\begin{array}{l}5 \cdot 24 \\
5 \cdot 69\end{array}$ & $\begin{array}{l}4 \cdot 33 \\
4 \cdot 36\end{array}$ & $\begin{array}{l}4 \cdot 78 \\
4 \cdot 97\end{array}$ \\
\hline 9. & $\begin{array}{l}4 \cdot 25 \\
4 \cdot 39\end{array}$ & $\begin{array}{l}4 \cdot 67 \\
4 \cdot 97\end{array}$ & $\begin{array}{l}4 \cdot 49 \\
4 \cdot 91\end{array}$ & $\begin{array}{l}5 \cdot 11 \\
5 \cdot 36\end{array}$ & $\begin{array}{l}5 \cdot 19 \\
5 \cdot 61\end{array}$ & $\begin{array}{l}5 \cdot 17 \\
5 \cdot 33\end{array}$ & $\begin{array}{l}5 \cdot 00 \\
5 \cdot 33\end{array}$ & $\begin{array}{l}4 \cdot 44 \\
4 \cdot 64\end{array}$ & $\begin{array}{l}4 \cdot 60 \\
4 \cdot 71\end{array}$ \\
\hline
\end{tabular}

The means of the two genotype replicates are presented in table 4 , and Hayman's analysis of variance in table 2 . The block interaction variances were homogenous $\left(\chi_{(5)}^{2}=3.9 ; \mathrm{P}=0.7\right)$ therefore all items of the main effects were tested against the common interaction term $(B t)$. There is significant additive variation between the populations (item $a$ ), also significant dominance variation (item $b$ ). All the components of $(b)$ are significant, the largest $\left(b_{1}\right)$, indicating the presence of directional dominance with lesser indications of gene asymmetry $\left(b_{2}\right)$ and inconsistent dominance interactions $\left(b_{3}\right)$. There is also a small $(c)$ item suggesting the presence of some slight consistent reciprocal differences. All of the individual block interactions except for $B \times b_{1}$ were significant when tested against the residual items, however, none of the replicate interactions were significant, neither were the main block and main replicate interactions, although the latter is large. It appears from this analysis that variation between blocks is of greater importance than that within genotypes.

The $W r / V r$ graph (fig. 2) shows a wide scatter of points which do not fall on any significant regression line; this indicates some form of non-allelic 
interaction. The distribution of points suggests that this may be of the complementary type. The correction of ordinate and abscissa for the environmental component $\left(E_{2}\right)$ reduces the level of dominance only slightly. The $W^{\prime} r / W r$ graph (fig. 2) does have a significant regression line in both replicates, these are not significantly different from each other, therefore, the joint regression is fitted $(b=0.316+0.090 ; \mathrm{P}=0.01)$.
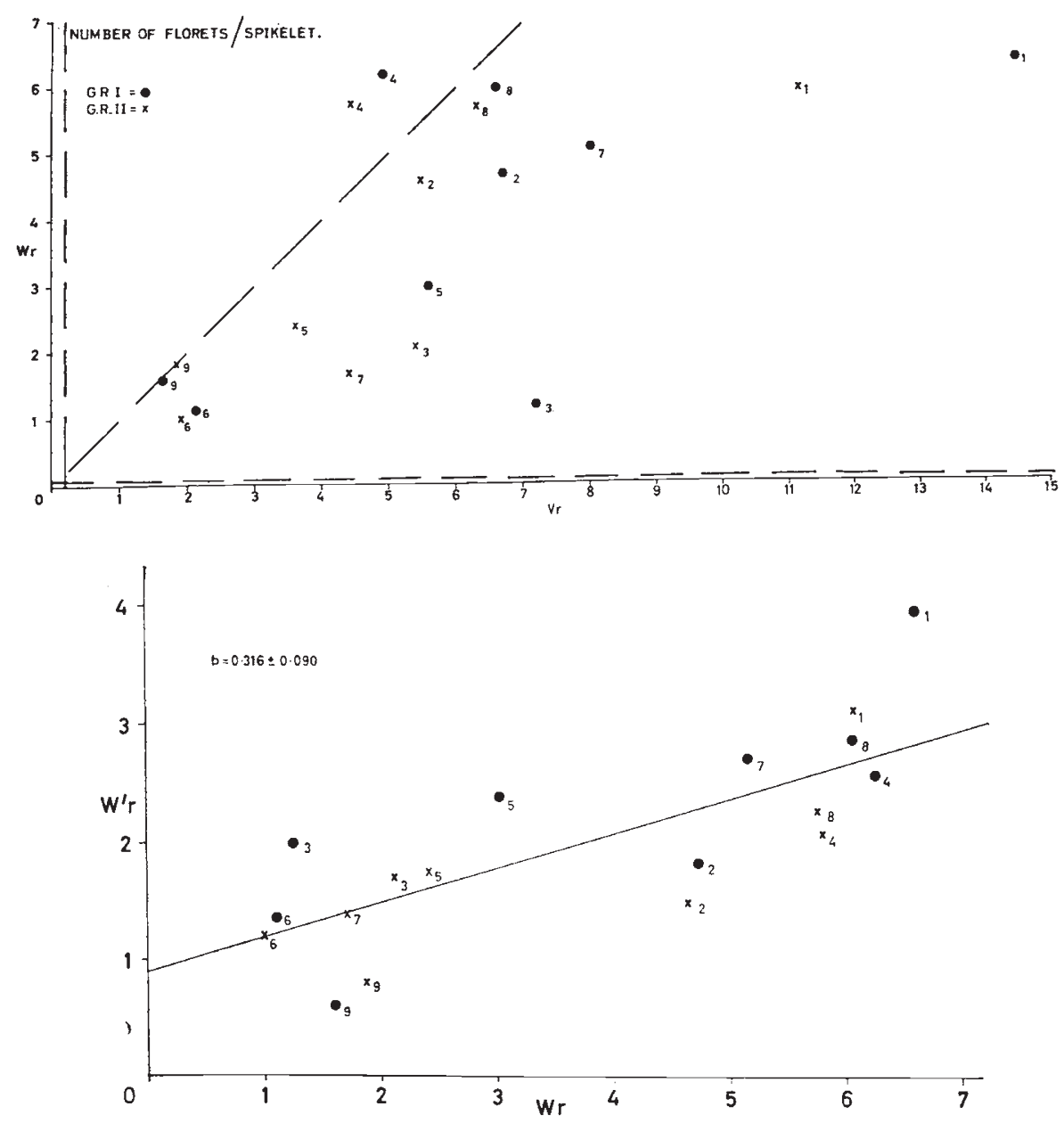

Fig. 2.- $W r / V r$ and $W^{\prime} r / W r$ graphs for number of florets per spikelet. Corrected ordinate and abscissa included as dashed lines.

Analysis of parental means (table 5a) shows that there are significant differences in the expression of additive genes between both replicates and blocks, with some interaction between them. The mean number of florets is different in the two replicates. The order of parental spikelet size is:

\section{Large}

G.R. I

G.R. II

53

3

$\begin{array}{ll}1 & 4 \\ 4 & 1\end{array}$

\begin{tabular}{ccccc}
\multicolumn{5}{c}{ Small } \\
6 & 9 & 7 & 8 & 2 \\
6 & 7 & 9 & 2 & 8
\end{tabular}


TABLE $5 a$

Analysis of variance of parental means

\begin{tabular}{lrcc}
\multicolumn{1}{c}{ Item } & D.F. & M.S. & V.R. \\
Parents & 8 & $2 \cdot 854$ & $30 \cdot 96^{* * *}$ \\
Blocks & 2 & $0 \cdot 417$ & $4 \cdot 52^{*}$ \\
Genotype replicates & 1 & $1 \cdot 047$ & $11 \cdot 36^{* *}$ \\
Blocks $\times$ Gen. reps. & 2 & $0 \cdot 263$ & $2 \cdot 85^{*}$ \\
Parents $\times$ Gen. reps. & 8 & $0 \cdot 059$ & - \\
Parents $\times$ blocks & 16 & $0 \cdot 143$ & - \\
Error & 16 & $0 \cdot 092$ & \\
Total & 53 & &
\end{tabular}

$$
* \mathrm{P}=0.05 ; * * \mathrm{P}=0.01 ; * * \mathrm{P}=0.001
$$

TABLE $5 b$

Analysis of variance of $\mathrm{Wr}$ and $\mathrm{Vr}$ for number of florets per spikelet

\begin{tabular}{lrrc}
\multicolumn{1}{c}{ Item } & D.F. & M.S. & V.R. \\
Genetic replicates & 1 & $36 \cdot 56$ & $5 \cdot 28^{*}$ \\
Arrays & 8 & $61 \cdot 77$ & $8 \cdot 92^{* * *}$ \\
Dominance & 1 & $260 \cdot 77$ & $37 \cdot 68^{* * *}$ \\
Genetic replicates $\times$ arrays & 8 & $7 \cdot 67$ & $1 \cdot 11$ N.S. \\
Genetic replicates $\times$ dominance & 1 & $1 \cdot 32$ & - \\
Arrays $\times$ dominance & 8 & 21.93 & $3 \cdot 17^{* *}$ \\
Gen. reps. $\times$ arrays $\times$ dominance & 8 & $1 \cdot 36$ & - \\
Error & 71 & $6 \cdot 92$ & \\
Total & 107 & &
\end{tabular}

$$
* \mathrm{P}=0.05 ; * * \mathrm{P}=0.01 ; * * \mathrm{P}=0.001
$$

Analysis of $W r$ and $V r$ (table $5 b$ ) shows that there is a significant mean square for genotype replicates, indicating a change in the proportion of dominant and recessive effects within the plant; the significance of the dominance $\times$ array mean square indicates that epistatic effects are important, these may also have contributed to the genotype replicate effects.

The ranking of the parents is:

\section{Dominant Recessive}

$\begin{array}{llllllllll}\text { G.R. I } & 1 & 7 & 8 & 2 & 4 & 5 & 3 & 6 & 9 \\ \text { G.R. II } & 1 & 4 & 2 & 8 & 3 & 7 & 5 & 9 & 6\end{array}$

(iii) Spikelet length

The data are presented in table 6, and Hayman's analysis of variance in table 2. Bartlett's test showed the block interactions to be homogeneous $\left(\chi_{(5)}^{2}=5.25 ; \mathrm{P}=0.7\right)$, therefore all the main items were tested against the common block interaction $(B t)$. There is significance additive variation between the parental populations (item $a$ ), also significant dominance variation (item $b$ ). The largest component of $(b)$ is $\left(b_{1}\right)$ indicating a directional dominance component, with some indications of gene asymmetry $\left(b_{2}\right)$, and inconsistent dominance interactions $\left(b_{3}\right)$, with this characte however, the more important genetic effects appear to be additive. 
The $W r / V r$ graph (fig. 3) shows the combined regression line $(b=$ $0.960 \pm 0.087 ; \mathrm{P}=0.001$ ), which cuts the $W r$ axis positively, this indicates that dominance is incomplete. The lack of correlation between the parental means and $(W r+V r)$ shows that this dominance must be ambi-directional.

TABLE 6

Mean spikelet length (mm.) for the first (top line) and second genetic replicates

\begin{tabular}{|c|c|c|c|c|c|c|c|c|c|}
\hline & 1 & 2 & 3 & 4 & 5 & 6 & 7 & 8 & 9 \\
\hline 1. & $\begin{array}{l}5 \cdot 12 \\
5 \cdot 03\end{array}$ & $\begin{array}{l}4 \cdot 85 \\
5 \cdot 16\end{array}$ & $\begin{array}{l}6.87 \\
7.05\end{array}$ & $\begin{array}{l}6.53 \\
6 \cdot 66\end{array}$ & $\begin{array}{l}7 \cdot 07 \\
7 \cdot 15\end{array}$ & $\begin{array}{l}6 \cdot 42 \\
6 \cdot 76\end{array}$ & $\begin{array}{l}6 \cdot 22 \\
6 \cdot 32\end{array}$ & $\begin{array}{l}6 \cdot 50 \\
6 \cdot 46\end{array}$ & $\begin{array}{l}5 \cdot 07 \\
5 \cdot 17\end{array}$ \\
\hline 2. & $\begin{array}{l}5 \cdot 19 \\
5 \cdot 26\end{array}$ & $\begin{array}{l}5 \cdot 30 \\
5 \cdot 41\end{array}$ & $\begin{array}{l}6 \cdot 39 \\
6 \cdot 78\end{array}$ & $\begin{array}{l}6 \cdot 53 \\
6 \cdot 76\end{array}$ & $\begin{array}{l}6 \cdot 58 \\
6 \cdot 62\end{array}$ & $\begin{array}{l}6 \cdot 53 \\
6 \cdot 82\end{array}$ & $\begin{array}{l}5 \cdot 67 \\
6 \cdot 05\end{array}$ & $\begin{array}{l}6 \cdot 10 \\
6 \cdot 35\end{array}$ & $\begin{array}{l}5 \cdot 60 \\
5 \cdot 86\end{array}$ \\
\hline 3. & $\begin{array}{l}6 \cdot 50 \\
6 \cdot 60\end{array}$ & $\begin{array}{l}6 \cdot 85 \\
7 \cdot 15\end{array}$ & $\begin{array}{l}6 \cdot 86 \\
7 \cdot 18\end{array}$ & $\begin{array}{l}7 \cdot 47 \\
7 \cdot 83\end{array}$ & $\begin{array}{l}7 \cdot 16 \\
7 \cdot 40\end{array}$ & $\begin{array}{l}6 \cdot 56 \\
6 \cdot 64\end{array}$ & $\begin{array}{l}6 \cdot 19 \\
6 \cdot 47\end{array}$ & $\begin{array}{l}7 \cdot 21 \\
7 \cdot 35\end{array}$ & $\begin{array}{l}6 \cdot 35 \\
6 \cdot 42\end{array}$ \\
\hline 4. & $\begin{array}{l}6 \cdot 31 \\
6 \cdot 66\end{array}$ & $\begin{array}{l}6 \cdot 25 \\
6 \cdot 46\end{array}$ & $\begin{array}{l}7.98 \\
8 \cdot 22\end{array}$ & $\begin{array}{r}7 \cdot 89 \\
8 \cdot 19\end{array}$ & $\begin{array}{l}7 \cdot 86 \\
8 \cdot 19\end{array}$ & $\begin{array}{l}6 \cdot 86 \\
7 \cdot 36\end{array}$ & $\begin{array}{l}6 \cdot 70 \\
6 \cdot 96\end{array}$ & $\begin{array}{l}6 \cdot 65 \\
6 \cdot 67\end{array}$ & $\begin{array}{l}6 \cdot 54 \\
6 \cdot 72\end{array}$ \\
\hline 5. & $\begin{array}{l}5 \cdot 91 \\
5 \cdot 96\end{array}$ & $\begin{array}{l}6 \cdot 70 \\
6 \cdot 93\end{array}$ & $\begin{array}{l}6 \cdot 65 \\
6 \cdot 88\end{array}$ & $\begin{array}{l}8 \cdot 08 \\
8 \cdot 11\end{array}$ & $\begin{array}{r}7 \cdot 80 \\
8 \cdot 08\end{array}$ & $\begin{array}{l}6 \cdot 71 \\
7 \cdot 01\end{array}$ & $\begin{array}{l}6.97 \\
7.09\end{array}$ & $\begin{array}{l}6.96 \\
7.08\end{array}$ & $\begin{array}{l}6 \cdot 40 \\
6 \cdot 61\end{array}$ \\
\hline 6. & $\begin{array}{l}6 \cdot 35 \\
6 \cdot 41\end{array}$ & $\begin{array}{l}6 \cdot 62 \\
6 \cdot 94\end{array}$ & $\begin{array}{l}7 \cdot 06 \\
7 \cdot 30\end{array}$ & $\begin{array}{l}7 \cdot 11 \\
7 \cdot 37\end{array}$ & $\begin{array}{l}6 \cdot 79 \\
7 \cdot 15\end{array}$ & $\begin{array}{l}6.53 \\
6.50\end{array}$ & $\begin{array}{l}6 \cdot 21 \\
6 \cdot 44\end{array}$ & $\begin{array}{l}6.74 \\
7.04\end{array}$ & $\begin{array}{l}5 \cdot 79 \\
5 \cdot 98\end{array}$ \\
\hline 7. & $\begin{array}{l}6 \cdot 32 \\
6 \cdot 51\end{array}$ & $\begin{array}{l}6 \cdot 10 \\
6 \cdot 18\end{array}$ & $\begin{array}{l}5 \cdot 83 \\
5 \cdot 95\end{array}$ & $\begin{array}{l}6 \cdot 59 \\
6 \cdot 79\end{array}$ & $\begin{array}{l}6 \cdot 56 \\
6 \cdot 62\end{array}$ & $\begin{array}{l}6 \cdot 37 \\
6 \cdot 68\end{array}$ & $\begin{array}{l}5 \cdot 01 \\
5 \cdot 12\end{array}$ & $\begin{array}{l}5 \cdot 85 \\
6 \cdot 15\end{array}$ & $\begin{array}{l}5 \cdot 97 \\
6 \cdot 17\end{array}$ \\
\hline 8. & $\begin{array}{l}6 \cdot 21 \\
6 \cdot 31\end{array}$ & $\begin{array}{l}6 \cdot 13 \\
6 \cdot 28\end{array}$ & $\begin{array}{l}7 \cdot 54 \\
7 \cdot 80\end{array}$ & $\begin{array}{l}6 \cdot 68 \\
6.88\end{array}$ & $\begin{array}{l}6.83 \\
6.97\end{array}$ & $\begin{array}{l}6 \cdot 75 \\
6.90\end{array}$ & $\begin{array}{l}5 \cdot 76 \\
6 \cdot 06\end{array}$ & $\begin{array}{l}5 \cdot 43 \\
5 \cdot 54\end{array}$ & $\begin{array}{l}6 \cdot 44 \\
6 \cdot 64\end{array}$ \\
\hline 9. & $\begin{array}{l}5 \cdot 15 \\
5 \cdot 32\end{array}$ & $\begin{array}{l}5 \cdot 83 \\
6 \cdot 02\end{array}$ & $\begin{array}{l}6 \cdot 20 \\
6 \cdot 64\end{array}$ & $\begin{array}{l}6 \cdot 64 \\
6 \cdot 94\end{array}$ & $\begin{array}{l}6 \cdot 57 \\
6 \cdot 91\end{array}$ & $\begin{array}{l}6 \cdot 50 \\
6 \cdot 62\end{array}$ & $\begin{array}{l}6 \cdot 05 \\
6 \cdot 48\end{array}$ & $\begin{array}{l}6 \cdot 18 \\
6 \cdot 39\end{array}$ & $\begin{array}{l}5.67 \\
5.84\end{array}$ \\
\hline
\end{tabular}

The $W^{\prime} r / W r$ graph (fig. 3 ) has a similar distribution of points and a regression $(b=0.349 \pm 0.073 ; P=0.001)$ which is significantly different from the theoretical line of $+0.5\left(t_{(7)}=0.01\right)$. This displacement of all the array points below the theoretical line suggests the occurrence of gene asymmetry.

Analysis of parental means (table 7a) shows that the parental genes have different additive effects which differ between replicates, but there are no significant differences in the expression of dominance effects between replicates, although there is a parents $\times$ blocks interaction which is highly significant, indicating differences of additive gene effects over blocks.

TABLE $7 a$

Analysis of parental means

\begin{tabular}{lrcc}
\multicolumn{1}{c}{ Item } & D.F. & M.S. & V.R. \\
Parents & 8 & $8 \cdot 362$ & $269 \cdot 74^{* * *}$ \\
Blocks & 2 & $0 \cdot 064$ & $2 \cdot 06$ N.S. \\
Genotype replicates & 1 & $0 \cdot 270$ & $8 \cdot 71 * *$ \\
Blocks $\times$ Gen. replicates & 2 & $0 \cdot 032$ & $1 \cdot 03$ N.S. \\
Parents $\times$ Gen. replicates & 8 & $0 \cdot 029$ & - \\
Parents $\times$ blocks & 16 & $0 \cdot 227$ & $7 \cdot 32 * * *$ \\
Error & 16 & $0 \cdot 031$ & \\
Total & 53 & \\
\multicolumn{2}{l}{$* \mathrm{P}=0 \cdot 05 ; * * \mathrm{P}=0 \cdot 01 ; * * \mathrm{P}=0 \cdot 001}$.
\end{tabular}



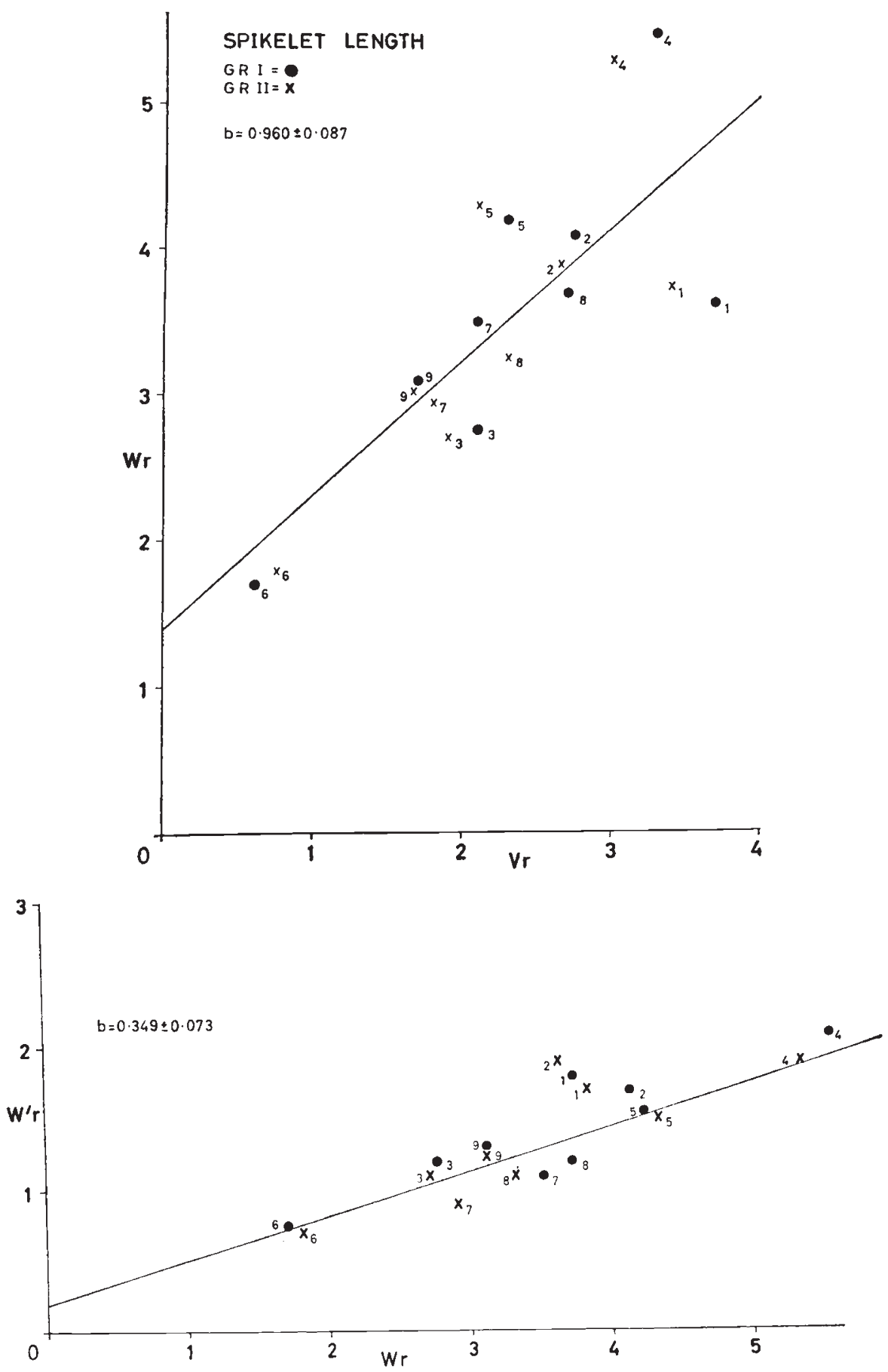

FIG. 3.- $W r / V r$ and $W^{\prime} r / W r$ graphs for spikelet length. 
TABLE $7 b$

Analysis of variance of $\mathrm{Wr}$ and $\mathrm{Vr}$ for spikelet length

\begin{tabular}{lrrc}
\multicolumn{1}{c}{ Item } & D.F. & M.S. & V.R. \\
Genetic replicates & 1 & $0 \cdot 445$ & - \\
Arrays & 8 & $9 \cdot 501$ & $7 \cdot 95^{* * *}$ \\
Dominance & 1 & $19 \cdot 746$ & $16 \cdot 53^{* * *}$ \\
Gen.reps. $\times$ arrays. & 8 & $0 \cdot 156$ & - \\
Gen.reps. $\times$ dom. & 1 & $0 \cdot 008$ & $-\overline{-}$ \\
Arrays $\times$ dom. & 8 & $1 \cdot 140$ & 0.95 N.S. \\
Gen.reps. $\times$ arrays $\times$ dom. & 8 & $0 \cdot 041$ & \\
Error & 71 & $1 \cdot 195$ & \\
Total & 107 & &
\end{tabular}

$$
{ }^{*} \mathrm{P}=0.05 ; * * \mathrm{P}=0.01 ;{ }^{* * * P}=0.001 \text {. }
$$

The analysis of $W r$ and $V r$ (table $7 b$ ) shows that there are differences in dominance between the parents (Arrays M.S.) also that mean dominance is not complete (Dominance M.S.). There are no significant differences in the expression of dominance effects between replicates.

\section{(jv) Lemma length}

Genotype replicates were not recorded in this, or the following characters, means are presented in table 8, and Hayman's analysis in table 9. Bartlett's

TABLE 8

Mean lemma length $(m m . \times 2)$

\begin{tabular}{|c|c|c|c|c|c|c|c|c|c|}
\hline & 1 & 2 & 3 & 4 & 5 & 6 & 7 & 8 & 9 \\
\hline 1. & 6.63 & $8 \cdot 30$ & 9.07 & $9 \cdot 60$ & $8 \cdot 78$ & 8.90 & 8.05 & $8 \cdot 48$ & 7.96 \\
\hline 2. & $8 \cdot 11$ & 8.83 & $9 \cdot 34$ & $10 \cdot 31$ & $9 \cdot 72$ & $10 \cdot 18$ & $9 \cdot 21$ & $9 \cdot 34$ & $9 \cdot 10$ \\
\hline 3. & $9 \cdot 89$ & $10 \cdot 19$ & $11 \cdot 39$ & $11 \cdot 60$ & $10 \cdot 84$ & $10 \cdot 73$ & $9 \cdot 55$ & $10 \cdot 67$ & $10 \cdot 78$ \\
\hline 4. & $9 \cdot 71$ & $10 \cdot 58$ & $11 \cdot 16$ & 12.64 & $11 \cdot 30$ & $11 \cdot 58$ & $10 \cdot 42$ & $10 \cdot 84$ & $10 \cdot 64$ \\
\hline 5. & $8 \cdot 17$ & $10 \cdot 54$ & $10 \cdot 97$ & $11 \cdot 78$ & 11.07 & $10 \cdot 85$ & $9 \cdot 86$ & $10 \cdot 22$ & $10 \cdot 00$ \\
\hline 6. & $9 \cdot 28$ & $10 \cdot 80$ & $10 \cdot 70$ & $11 \cdot 72$ & $10 \cdot 90$ & 10.94 & $9 \cdot 39$ & $10 \cdot 61$ & $10 \cdot 47$ \\
\hline 7. & $7 \cdot 90$ & $9 \cdot 72$ & $9 \cdot 36$ & $10 \cdot 49$ & $9 \cdot 44$ & $9 \cdot 56$ & 7.96 & $9 \cdot 08$ & $9 \cdot 08$ \\
\hline 8. & $8 \cdot 33$ & $9 \cdot 83$ & $10 \cdot 82$ & $10 \cdot 96$ & $9 \cdot 75$ & $10 \cdot 46$ & $9 \cdot 00$ & 8.88 & 10.37 \\
\hline 9. & 8.42 & $9 \cdot 55$ & $10 \cdot 30$ & $10 \cdot 80$ & $10 \cdot 17$ & $10 \cdot 31$ & $9 \cdot 34$ & $10 \cdot 83$ & $9 \cdot 53$ \\
\hline
\end{tabular}

test shows the error mean square to be homogeneous $\left(\chi_{(5)}^{2}=4.5 ; P=0.5\right)$, therefore all items were tested against the common error item $(B t)$. There are significant additive genetic differences between the parental populations (item $a$ ) also a significant dominance component (jtem $b$ ). The $(c)$ item indicating the presence of consistent reciprocal differences is also significant. The size of the $(a)$ item suggests however that most of the genetic variation is additive. 
TABLE 9

Hayman's analysis of variance for lemma length

\begin{tabular}{|c|c|c|c|}
\hline Item & D.F. & M.S. & V.R. \\
\hline$a$ & 8 & $32 \cdot 586$ & $187 \cdot 27 * * *$ \\
\hline$b_{1}$ & 1 & $0 \cdot 671$ & 3.86 N.S. \\
\hline$b_{2}$ & 8 & $0 \cdot 843$ & $4 \cdot 84 * * *$ \\
\hline$b_{3}$ & 27 & 0.517 & $2 \cdot 97^{* *}$ \\
\hline$b$ & 36 & 0.594 & $3 \cdot 41 * * *$ \\
\hline$c$ & 8 & 0.655 & $3 \cdot 76^{* *}$ \\
\hline$d$ & 28 & $0 \cdot 114$ & 0.65 N.S. \\
\hline Total & 80 & & \\
\hline$B$ & 2 & 0.395 & $2 \cdot 27$ \\
\hline$B t$ & 160 & $0 \cdot 174$ & \\
\hline Total & 242 & & \\
\hline
\end{tabular}

The $W r / V r$ graph (fig. 4$)$ is fitted with a joint regression line $(b=$ $1 \cdot 051 \pm 0 \cdot 168 ; \mathrm{P}=0 \cdot 001)$ as the reciprocal regressions are not significantly different $(P=0 \cdot 20)$. The regression cuts the $W r$ axis positively, indicating incomplete dominance. The lack of correlation between the parental means and $(W r+V r)$ shows this to be ambidirectional. The $W^{\prime} r / W r$ graph also shows a similar distribution of parental points, the regression $(b=0.0373 \pm$ $0.041 ; \mathrm{P}=0.001)$ is significantly different from $+0.5\left(t_{(7)}=0.01\right)$.

\section{(v) Awn length}

The means are presented in table 10 and Hayman's analysis in table 11. The error mean squares are homogenous $\left(\chi_{(5)}^{2}=4.7 ; P=0.5\right)$, therefore all items were tested against the common error $(B t)$. There is significant additive genetic variation between the populations (item $a$ ), also significant dominance variation (item $b$ ). All the components of $(b)$ are significant,

TABLE 10

Mean awn length $(m m . \times 2)$

$\begin{array}{lccccccccc} & 1 & 2 & 3 & 4 & 5 & 6 & 7 & 8 & 9 \\ 1 . & 0.64 & 1.12 & 1.06 & 1.13 & 0.95 & 0.93 & 0.82 & 0.76 & 0.80 \\ \text { 2. } & 1.07 & 1.38 & 1.20 & 1.08 & 1.17 & 1.41 & 1.41 & 1.17 & 1.19 \\ \text { 3. } & 1.19 & 1.32 & 1.58 & 1.28 & 1.25 & 1.38 & 1.11 & 1.20 & 1.34 \\ \text { 4. } & 0.98 & 1.18 & 1.45 & 1.22 & 1.05 & 1.05 & 1.12 & 0.96 & 1.11 \\ \text { 5. } & 0.72 & 1.48 & 1.20 & 1.22 & 1.24 & 1.17 & 1.22 & 1.01 & 1.05 \\ 6 . & 1.09 & 1.61 & 1.35 & 1.01 & 1.24 & 1.44 & 1.07 & 0.84 & 1.22 \\ 7 . & 0.75 & 1.48 & 1.12 & 1.19 & 1.03 & 1.00 & 0.97 & 1.05 & 1.11 \\ \text { 8. } & 0.68 & 1.34 & 1.26 & 1.02 & 0.92 & 1.13 & 1.02 & 1.43 & 1.32 \\ \text { 9. } & 0.89 & 1.24 & 1.22 & 1.19 & 1.15 & 1.17 & 1.17 & 1.37 & 0.96\end{array}$

the largest $\left(b_{1}\right)$ indicating the presence of directional dominance, $\left(b_{2}\right)$ gene asymmetry, and $\left(b_{3}\right)$ inconsistent dominance interactions. There is also a small $(c)$ item suggesting the presence of some consistent reciprocal effects. 

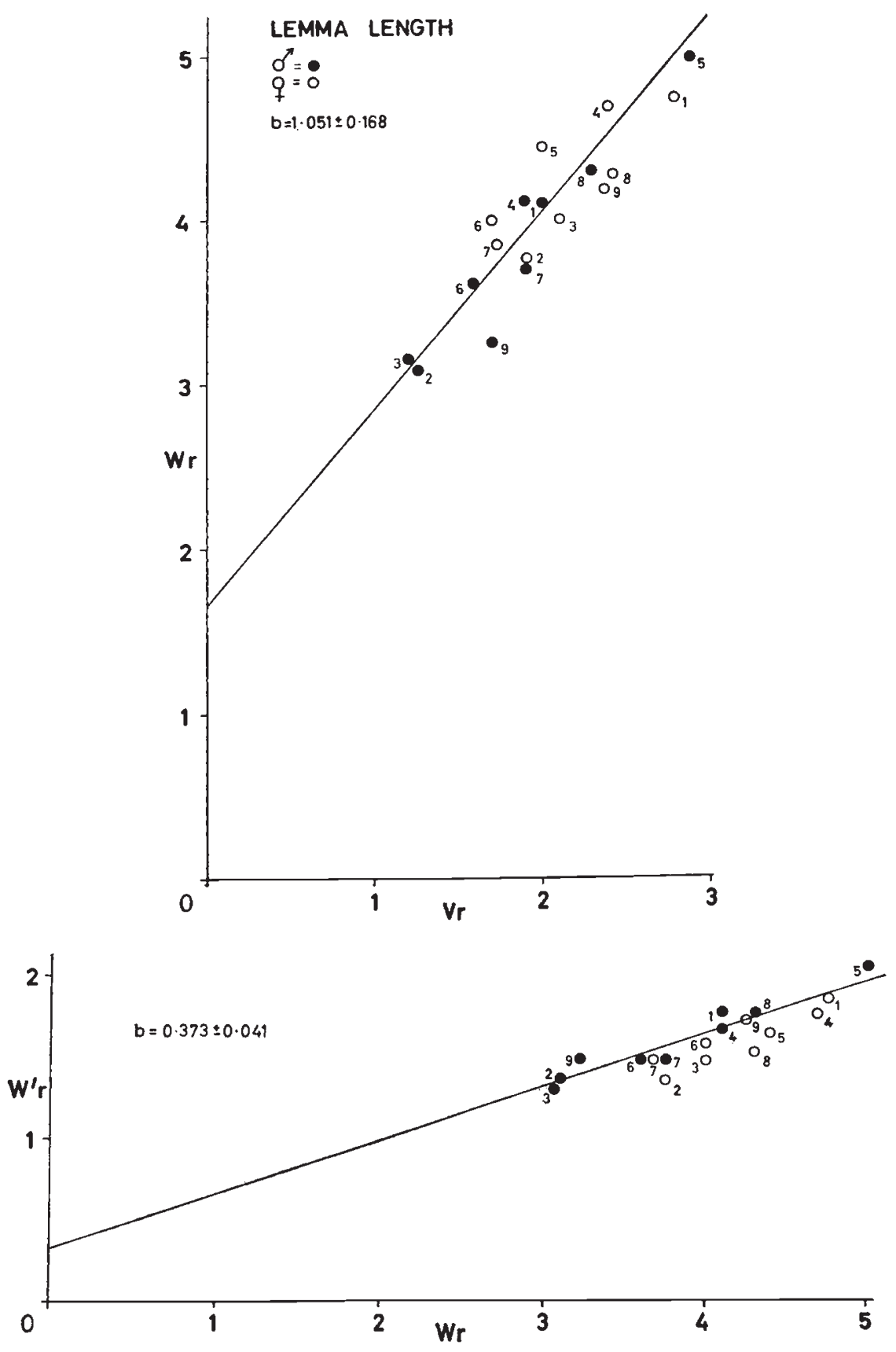

FIG. 4. $-W r / V r$ and $W^{\prime} r / W r$ graphs for lemma length. 
TABLE 11

Hayman's analysis of variance for awn length

\begin{tabular}{|c|c|c|c|}
\hline Item & D.F. & M.S. & V.R. \\
\hline$a$ & 8 & $0 \cdot 720$ & $42 \cdot 35^{* * * *}$ \\
\hline$b_{1}$ & 1 & $0 \cdot 133$ & $7 \cdot 82^{* *}$ \\
\hline$b_{2}$ & 8 & $0 \cdot 101$ & $5.94^{* * *}$ \\
\hline$b_{3}$ & 27 & $0 \cdot 080$ & $4 \cdot 70^{* * *}$ \\
\hline$b$ & 36 & $0 \cdot 086$ & $5 \cdot 06^{* * *}$ \\
\hline$c$ & 8 & 0.034 & $2 \cdot 00^{*}$ \\
\hline$d$ & 28 & $0 \cdot 022$ & 1.29 N.S \\
\hline Total & 80 & & \\
\hline$B$ & 2 & $0 \cdot 005$ & \\
\hline$B t$ & 160 & $0 \cdot 017$ & \\
\hline Total & 242 & & \\
\hline
\end{tabular}

The $W r / V r$ graph (fig. 5) shows no significant regression, both male and female points are scattered across the graph in a random manner, indicating that the disturbance is due to non-allelic interaction. The correction of the ordinate and abscissa for the environmental component $\left(E_{2}\right)$ reduces the level of dominance expressed only slightly. The $W^{\prime} r / W r$ graph which is relatively undisturbed by non-allelic interaction, shows a significant joint regression $(b=0.316 \pm 0.108 ; P=0.05)$, with the array points of castellata (9) and lusitanica (3) some distance below the theoretical line, suggesting that these two populations have genes not present in the other samples.

\section{(vi) Lemma apex}

The character is portrayed in figure 6, means are presented in table 12, and Hayman's analysis in table 13. Each main item was tested against its own error mean square, as these were heterogeneous $\left(\chi_{(5)}^{2}=39.4\right.$; $\mathrm{P}=0.001)$. There is significant additive genetic variation between the parental populations (item $a$ ), also significant dominance variation (item $b$ ). Only two components of dominance are significant, those indicating gene asymmetry $\left(b_{2}\right)$ and inconsistent dominance interactions $\left(b_{3}\right)$. Item $(d)$ is also significant, indicating the presence of inconsistent reciprocal differences.

TABLE 12

\begin{tabular}{lccccccccc}
\multicolumn{10}{c}{ Lemma apex notch $(\mathrm{mm} . \times 2)$} \\
1. & 1 & $\mathbf{0}$ & 3 & 4 & 5 & 6 & 7 & 8 & 9 \\
2. & 0.035 & 0.140 & 0.193 & 0.107 & 0.146 & 0.088 & 0.164 & 0.108 & 0.257 \\
3. & 0.204 & 0.330 & 0.295 & 0.084 & 0.257 & 0.150 & 0.174 & 0.219 & 0.204 \\
4. & 0.064 & 0.064 & 0.257 & 0.208 & 0.174 & 0.285 & 0.215 & 0.226 & 0.240 \\
5. & 0.244 & 0.208 & 0.211 & 0.058 & 0.196 & 0.076 & 0.200 & 0.114 & 0.149 \\
6. & 0.035 & 0.264 & 0.222 & 0.065 & 0.060 & 0.122 & 0.096 & 0.137 & 0.096 \\
7. & 0.185 & 0.219 & 0.210 & 0.044 & 0.249 & 0.098 & 0.236 & 0.169 & 0.314 \\
8. & 0.088 & 0.185 & 0.254 & 0.047 & 0.133 & 0.143 & 0.203 & 0.460 & 0.262 \\
9. & 0.160 & 0.235 & 0.250 & 0.104 & 0.159 & 0.097 & 0.237 & 0.280 & 0.199
\end{tabular}



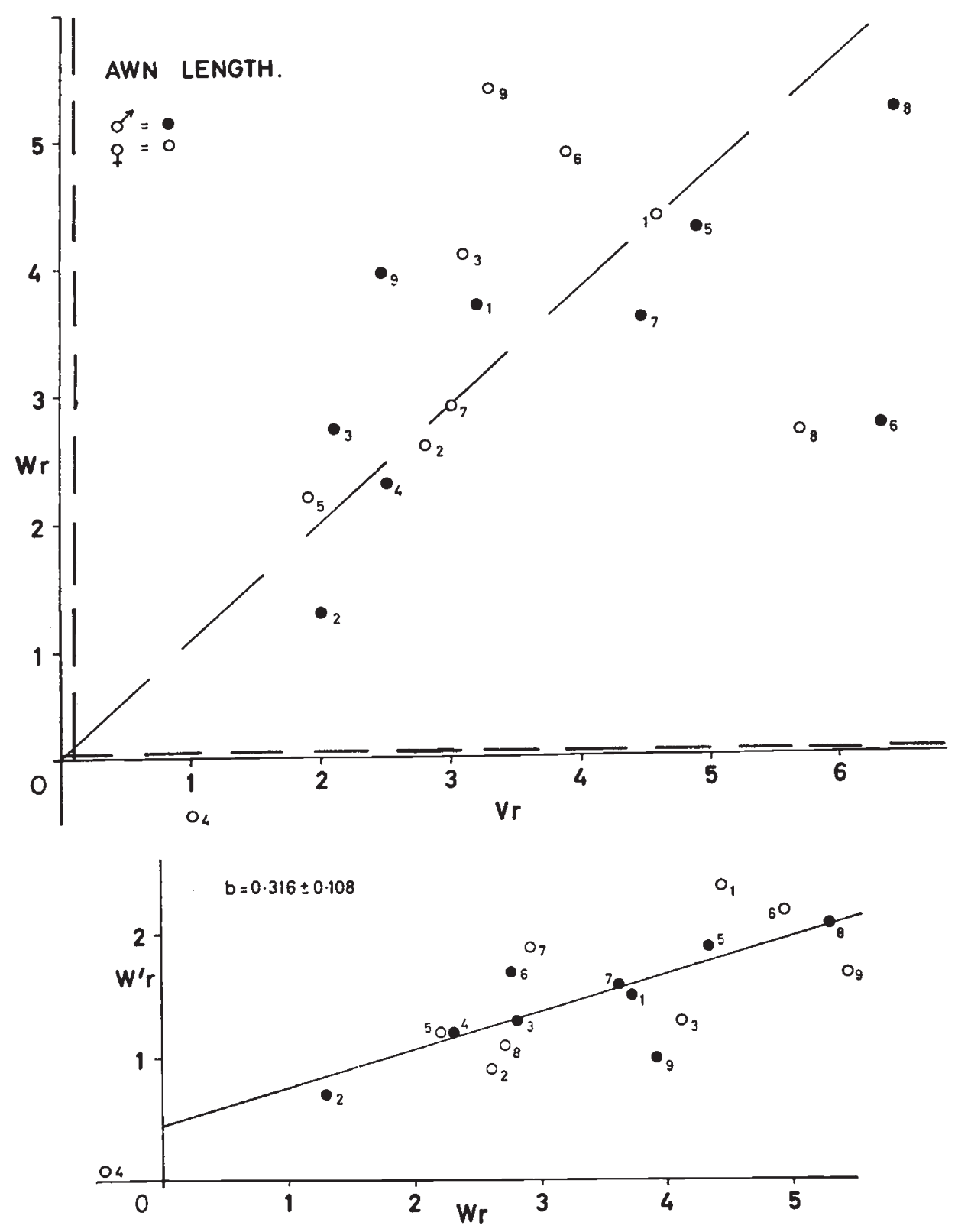

FIG. 5.- $W r / V r$ and $W^{\prime} r / W r$ graphs for awn length, corrected ordinate and abscissa included as dashed lines. 
TABLE 13

Hayman's analysis of lemma apex notch

$\begin{array}{crcc}\text { Item } & \text { D.F. } & \text { M.S. } & \text { V.R. } \\ a & 8 & 0.1164 & 64 \cdot 31^{* * *} \\ b_{1} & 1 & 0.0617 & 9 \cdot 82 \mathrm{~N} . \mathrm{S} . \\ b_{2} & 8 & 0.0294 & 13.86^{* * *} \\ b_{3} & 27 & 0.0111 & 3.97 * * * \\ b & 36 & 0.0166 & 6 \cdot 04^{* * *} \\ c & 8 & 0.0009 & 0.57 \mathrm{~N} . \mathrm{S} \\ d & 28 & 0.0036 & 2 \cdot 30^{* *} \\ \text { Total } & 80 & & \\ & & & \\ \text { Block } & 2 & 0.0009 & \\ B a & 16 & 0.0018 & \\ B b_{1} & 2 & 0.0063 & \\ B b_{2} & 16 & 0.0021 & \\ B b_{3} & 54 & 0.0028 & \\ B b & 72 & 0.0027 & \\ B c & 16 & 0.0016 & \\ B d & 56 & 0.0016 & \\ \text { Total } & 242 & & \\ & & & \\ * \mathrm{P}=0.05 ; * \mathrm{P}=0.01 ; * * * \mathrm{P}=0.001 .\end{array}$

The $W r / V r$ graph (fig. 7) shows a significant joint regression $(b=$ $0.956 \pm 0.118 ; P=0.001)$, which cuts the axes at their intersection, this indicates full dominance for the character. There is no correlation between the parental means and $(W r+V r)$ therefore dominance is ambi-directional.
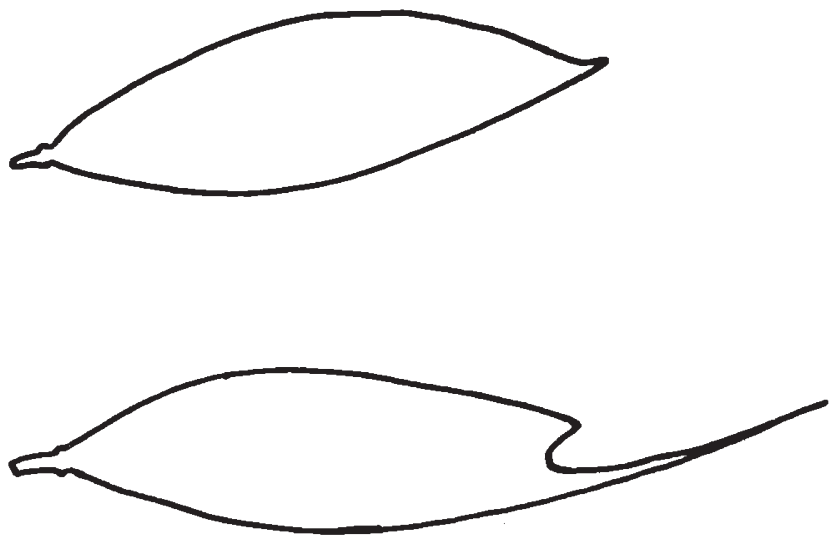

Fig. 6.-Lemma, showing apex forms.

The population of subspecies sample santai (8) stands out as having the highest number of recessive genes, also the deepest apical lobe, the rest of the population points are concentrated toward the lower (dominant) end of the graph. The $W^{\prime} r / W r$ graph (fig. 7) shows a similar distribution of points, the regression line $(b=0.218 \pm 0.015 ; \mathrm{P}=0.001)$ deviates significantly from the theoretical $\left(t_{7}=0.001\right)$, this is caused entirely by the population 
sample of santai (8), which indicates that the population has a large number of genes not distributed throughout the other population samples considered.
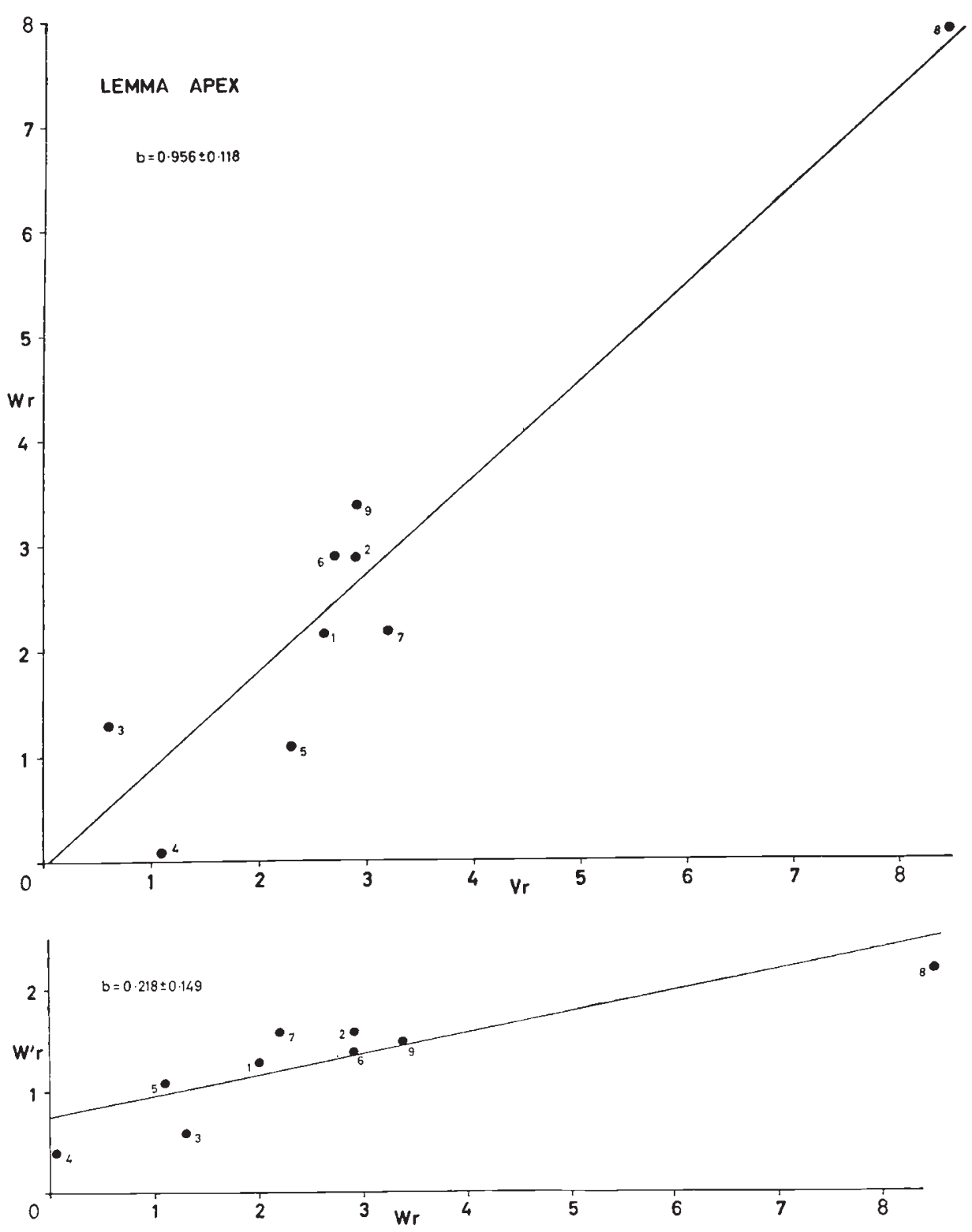

Fig. 7.- $W r / V r$ and $W^{\prime} r / W r$ graphs for lemma apex.

\section{Discussion}

(i) Stability of genetic replicates

The data for panicle length show that there is a significant variation of additive gene expression in the parental populations in successively produced 
heads, caused by micro-environmental and developmental effects. This variability is reflected in the response of the progeny of these particular parents, which from their position on the $W r / V r$ graphs appear to be the populations containing the more heterozygous and recessive gene combinations, i.e. stability tends to be dominant as shown by Paxman (1956) and Broadhurst and Jinks (1966). The presence of morphological variability did not however, alter the overall level of dominance in the arrays, only the relative expression of the proportion of dominant and recessive genes in the parents concerned, depending upon the panicle selected. The degree of dominance expressed is incomplete, and directional for short panicles. This is not a geographical trend, for although some northern populations do have longer panicles, this is only one aspect of floral adaptation to a cooler, damper, climate.

The number of florets per spikelet also show differences due to different environmental effects and/or developmental variations occurring between the two genotype replicates, these differences are not very great in the parental populations, but assume more importance in the $F_{1}$ progenies, particularly those of ibizensis (7) suggesting either a disturbed polygenic balance or a more rapid rate of differentiation for this character. The distribution of the array points of the two genotype replicates indicates that considerable non-allelic interaction, probably of the complementary type, is occurring, giving a pattern of spurious overdominance. Spikelet length shows no significant within plant differences of gene expression, and the pattern of inheritance is also much easier to interpret than that of the previous character. Dominance is incomplete and ambi-directional, indicating that. selection has presumably been favouring an intermediate optimum in each population, although it is possible that dominance could be in opposite directions in different populations giving an over-all picture of ambi-directional dominance when they are crossed.

In the characters discussed above, the stability of gene expression, i.e. the response to fluctuations of both the internal and external environment, varies between different populations. This physiological developmental threshold determining gene expression within the individual also differs with the characters concerned; in Dactylis therefore, as in Lycopersicum esculentum (Williams, 1960) and Nicotiana rustica (Paxman, 1956), this variation is specific to individual characters in relation to particular environmental influences. Both the above authors showed that unrelated characters within a plant had unrelated stabilities; Paxman (1956) also showed that in Nicotiana, characters closely related developmentally (flower parts) did possess related stabilities. This is not so in Dactylis with regard to the two spikelet characters, for mean floret numbers varies significantly, appearing to experience competition (probably due to density effects) depending upon position in the panicle, while mean spikelet size does not show this effect.

\section{(ii) Inheritance pattern of correlated characters}

Table 14 shows the phenotypic correlations between the characters studied within the nine parental populations. There is a large and highly significant correlation between the number of florets and spikelet length in most populations, and weak correlations between spikelet length and lemma length, and the number of florets and lemma length in the populations of 
santai (8) and castellata (9). Whereas the $W r / V r$ graph for number of florets shows some form of non-allelic interaction, spikelet length shows incomplete dominance, however the scatter of points on the two graphs is similar, and the developmental association could be caused either by pleiotropism or linkage. If the latter is the case, then this linkage has been selected against in the population of parthiana (5) where there is no statistically significant relationship.

The pattern of inheritance for lemma length shows incomplete and ambidirectional dominance, with a large consistent difference between the reciprocals. The positive phenotypic correlation between lemma and awn length is present only in two-thirds of the populations used, and is apparently an ecological rather than a developmental relationship, as the populations lacking this correlation may have either very short or very long awns.

TABLE 14

Intra-population correlations for the parents

$\begin{array}{lccccccccc}\begin{array}{l}\text { Populations } \\ \text { Characters }\end{array} & 1 & 2 & 3 & 4 & 5 & 6 & 7 & 8 & 9 \\ \begin{array}{l}\text { Panicle L./ } \\ \quad \text { Spikelet L. }\end{array} & -0.02 & -0.57^{*} & -0.34 & 0.42 & 0.20 & -0.06 & 0.41 & -0.38 & -0.20 \\ \begin{array}{c}\text { Panicle L./ } \\ \text { Lemma L. }\end{array} & -0.06 & 0.53^{*} & -0.15 & 0.70^{* * *} & 0.09 & 0.12 & -0.14 & -0.07 & 0.23 \\ \begin{array}{c}\text { Panicle L./ } \\ \text { Awn L. }\end{array} & -0.43 & 0.65^{* *} & -0.03 & 0.08 & 0.03 & 0.10 & 0.01 & -0.08 & 0.04 \\ \begin{array}{c}\text { No. Florets/ } \\ \text { Spikelet L. }\end{array} & 0.90^{* * *} & 0.91^{* * *} & 0.85^{* * *} & 0.79^{* * *}-0.28 & 0.62^{* *} & 0.77^{* * *} & 0.96^{* * *} & 0.75^{* * *} \\ \begin{array}{c}\text { No Florets/ } \\ \text { Lemma L. }\end{array} & 0.13 & -0.48^{*} & 0.11 & 0.17 & 0.27 & 0.42 & 0.46 & 0.47^{*} & 0.47^{*} \\ \begin{array}{c}\text { No. Florets/ } \\ \text { Awn L. }\end{array} & 0.30 & -0.49^{*} & 0.02 & 0.14 & -0.21 & 0.31 & -0.08 & 0.24 & 0.43 \\ \begin{array}{c}\text { Spikelet L./ } \\ \text { Lemma L. }\end{array} & 0.41 & -0.44 & 0.07 & 0.29 & 0.22 & 0.42 & 0.38 & 0.49^{*} & 0.53^{*} \\ \begin{array}{c}\text { Lemma L./ } \\ \text { Awn L. }\end{array} & 0.12 & 0.63^{* *} & 0.24 & 0.54^{*} & 0.55^{*} & 0.65^{* *} & 0.53^{*} & 0.78^{* * *} & 0.26\end{array}$

There is also no relation between the genetic systems controlling lemma and awn length, the pattern of inheritance of awn length is disturbed by both consistent reciprocal differences and non-allelic interaction, however, the probability is that the interaction is of the duplicate type. The lemma apex character, which was included because of its emphasis in some classifications of the genus, appears to be simply inherited and shows full dominance. Selection has been stabilising and most genes for deeply notched apex, which are recessive, are asymmetrically distributed in population 8 (santai).

Little direct evidence for the genetic control of correlated characters is available, although Cooper (1960) concluded from his study of two populations of Lolium perenne, that linkage was responsible. It is possible that both pleiotropic gene effects and linkage of polygenic systems controlling different characters are effective in Dactylis, although linked polygenes are probably the more likely explanation (Mather, 1943).

\section{(iii) Conclusions}

The characters considered above, and in the previous papers (Parker, $1968 a, b)$ were selected for an experimental study in breadth to analyse the 
nature of genetic differences between divergent populations within a species. The various subspecies populations of Dactylis can be distinguished morphologically, but analysis of hybrids shows that they cannot be consistently and clearly distinguished in genetical terms with regard to characters other than those closely associated with reproductive fitness. Fisher's fundamental theorem of natural selection states that the rate of change of fitness in a population is proportional to the additive genetic variance remaining in it (Fisher, 1930). Therefore the population which has reached equilibrium with its present environment should have little or no additive genetic variance in characters closely connected with fitness, although genotypes may vary

TABLE 15

Expressions of dominance, cytoplasmic effects and nuclear/cytoplasmic interactions for all characters used in the study. $\mathrm{c}$ only $=\mathrm{c}$ significant; $\mathrm{c}=\mathrm{d}, \mathrm{c}$ and $\mathrm{d}$ equally significant; $\mathrm{c}>\mathrm{d}, \mathrm{c}$ is significantly greater than $\mathrm{d}$, but both are significant

\section{Character}

1. Seed set

2. Seed germination

3. Fertility index

4. Growth rate

5. Internode length

6. Leaf six area

7. Tiller number

8. Emergence date

9. Flag leaf area

10. Summer leaf area

11. Leaf dry weight

12. Chlorophyl content (a) Chlorophyl content $(b)$

13. Panicle length

14. Floret number

15. Spikelet length

16. Lemma length

17. Awn length

18. Lemma apex
$W r / V r$ Analysis

Spurious overdominance, gene asymmetry Spurious overdominance, gene asymmetry Directional overdominance, gene asymmetry Complementary gene interaction

Incomplete directional dominance

Incomplete directional dominance; some interaction

Incomplete ambi-directional dominance

Incomplete directional dominance

Incomplete ambi-directional dominance

Incomplete directional dominance

Gene interaction

Incomplete ambi-directional dominance

Incomplete ambi-directional dominance

Incomplete directional dominance

Complementary gene interaction

Incomplete ambi-directional dominance

Incomplete ambi-directional dominance

Duplicate gene interaction

Complete ambi-directional dominance; gene asymmetry

Hayman's
Analysis
$\mathrm{F}_{1} c ; \mathrm{F}_{2}-$
$\mathrm{F}_{1} c ; \mathrm{F}_{2}-$
$\mathrm{F}_{1} c ; \mathrm{F}_{2}-$
$c=d$
$c=d$
$c$ only
$c=d$
$c=d$
$c=d$
$c$ only
$c$ only
$c>d$
$c>d$
$c$ only
$c$ only
$c$ only
$d$ only

Hayman's $\mathrm{F}_{1} c ; \mathrm{F}_{2}$ $\mathrm{F}_{1} c ; \mathrm{F}_{2}-$ $\mathrm{F}_{1} c ; \mathrm{F}_{2}-$ $c=d$

$c=d$

$c$ only

$c=d$

$c=d$

$c$ only

$c$ only

$c>d$

$c$ only

$c$ only

d only

with regard to their over-all fitness. On the other hand, where moderate changes of the phenotype can have no effect on reproductive fitness, the hereditary variance will be almost entirely additive, the results (table 15) show, within experimental limits, that this occurs in Dactylis. The characters concerned with fertility (nos. 1, 2, 3, table 15) present a pattern of genetic variation expressing spurious overdominance. In the interpopulation hybrids there is obviously considerable disturbance of the relational balance of the polygenic systems controlling fertility, which appear to be independently balanced, with natural selection acting directionally towards high viability within each population. Some other characters of apparent importance with regard to viability and fertility of the parental populations in the different ecological niches they occupy, such as growth rate, number of florets, and awn length (nos. 4, 14, 17, table 15) also show gene interaction and dominance. The effect of natural selection has been to change these characters in different directions, being initially disruptive, then stabilising towards 
the optimum favoured in each environment. This has resulted in the present genetic polymorphism. The remaining eleven characters under consideration are controlled by polygenic systems of low dominance, and low interaction, on which natural selection has acted sometimes in a stabilising manner, sometimes directionally, these characters apparently have only a slight effect on viability.

The large amount of consistent and inconsistent interaction which occurs throughout most of the analyses is of interest, for the parents although perennials, reproduce by seed and live in fairly open or disturbed habitats. There is clearly strong selection for mutual balance of parental genes and cytoplasm in each environment, for both consistent and inconsistent reciprocal effects are apparent in the $F_{1}$ plants through to the adult stage. Analysis of the seed characters (Parker, 1968a), also analysis of some other characters in the $\mathrm{F}_{2}$ generation (Parker, unpub.), show that these effects do not go beyond the $\mathrm{F}_{1}$.

The consistent reciprocal effect is dependent on differences in the embryonic environment which are probably due to maternal nuclear products in the cytoplasm, the other effect is a differential interaction between the $F_{1}$ hybrid nucleus and the parental cytoplasms in various crosses. Consistent effects in the $F_{1}$ are a fairly common phenomenon, not only in hybrids between reproductively isolated populations, but also hybrids in sexually reproduced perennial cultivars such as Lucerne (Lamprecht, 1964), they are not however expressed by all individual characters, some genes are apparently insensitive to the cytoplasmic milieu. This also applies to the analysis of within-plant variability, this shows clearly that in some characters only, and in some parents only, there was an environmental effect which was largely one of increasing or reducing the differences in expression of dominance of the various genes, this variation is presumably adaptive in the original habitats of the populations concerned. This study shows that large, usually nonallelic, genetic differences are found only where the measured characters are apparently directly involved with viability and "fitness". Thus these spatially isolated populations have accumulated adaptive genetic differences often as separate components of sets of dominant genes with complementary and duplicate interactions, and it is probable that these interactions are also the cause of the incipient reproductive isolation observed (Parker and Borrill, 1968). It is apparent that the stresses of natural selection on the various major morphological and physiological characters of the nine subspecies populations have, during the period of their isolation, considerably altered their fundamental genetic characteristics.

\section{Summary}

1. The investigation of the genetic variation present in some populations of diploid Dactylis was concluded with a study of panicle and floret characters.

2. Analysis of the hybrids showed that the pattern of variation was largely additive, with some characters controlled by polygenic systems characterised by large amounts of non-allelic interaction.

3. Analysis of genotype replicates showed that some characters were affected by non-heritable variation to a certain degree.

4. The results are discussed, and some conclusions drawn from the complete set of analyses. 
Acknowledgments.--This work was carried out at the Welsh Plant Breeding Station during the tenure of a Ministry of Agriculture scholarship, and submitted in partial fulfillment of a Ph.D. thesis. I wish to thank Professor P. T. Thomas for his general guidance and interest in the thesis. I also wish to express my thanks to Professor J. L. Jinks for his subsequent discussion of points in the manuscript, and his advice on the modification of Hayman's analysis used here.

\section{REFERENCES}

ALLARD, R. W. 1956. The analysis of genetic-environmental interactions by means of diallel crosses. Genetics, 41, 305-318.

BROADHURST P. L., AND JINKS J. L. 1966. Stability and change in the inheritance of behaviour in rats: a further analysis of statistics from a diallel cross. Proc. Roy. Soc. B., 165, 450-472. COOPER, J. P. 1960. Selection and population structure in Lolium. IV. correlated response to selection. Heredity, 14, 229-246.

FISHER, R. A. 1930. The genetical theory of natural selection. O.U.P., $268 \mathrm{pp}$.

HAYMAN, B. I. 1958. Theory and analysis of diallel crosses II. Genetics, 43, 63-85.

JiNKs, J. L. 1954. The analysis of continuous variation in a diallel cross of Nicotiana rustica varieties. Genetics, 34, 767-788.

LAMPRECHT M. P., AND STEVENS J. M. 1964. A genetic study of certain characteristics of the lucerne cultivar "South African Standard" (Medicago sativa L.). S. Afr. Tydskrif. Landbouwet, 7, 749-762.

MAther, K. 1943. Polygenic inheritance and natural selection. Biol. Rev., 18, 32-64.

MATHER, K. 1967. Complementary and duplicate gene interactions in biometrical genetics. Heredity, 21, 97-103.

PARKER, P. F. 1968a. Genetic studies in Dactylis. I. Cross compatibility and seedling characters. Heredity, $23(2), 279-300$.

PARKer, P. F. 1968b. Genetic studies in Dactylis. II. Emergence date, and some morphological and physiological leaf characters. Heredity, 23 (3), (in press).

PARKER, P. F., AND BORRILL, M. 1968. Studies in Dactylis. I. Fertility relationships in some diploid subspecies. New Phytol., 67, 649-662 (in press).

Paxman, G. J. 1956. Differentiation and stability in the development of Nicotiana rustica. Ann. Bot. N.S., $20(78), 331-347$.

Williams w. T. 1960. Relative variability of inbred lines and $\mathrm{F}_{1}$ hybrids in Lycopersicum esculentum. Genetics, 45, 1457-1465. 